\title{
Análise dos Métodos de Avaliação, dos Recursos e do Reconhecimento da Fisioterapia Oncológica nos Hospitais Públicos do Distrito Federal
}

\author{
Analysis of Evaluation Methods, Resources and Recognition of Oncologic \\ Physiotherapy in Distrito Federal Public Hospitals
}

\author{
Carla Andréa Machado Borges ${ }^{1}$, Caroline de Faria Silveira ${ }^{2}$, Paulo Cezar Menezes Tavares Lacerda ${ }^{1}$, Marinha Tereza Alves Nascimento ${ }^{3}$
}

\begin{abstract}
Resumo
Introdução: Câncer é o nome dado a um grupo de doenças malignas caracterizadas pelo crescimento celular anormal e que podem espalhar-se para várias regiōes do corpo. Apesar de ser responsável anualmente por $12,5 \%$ das mortes no mundo, ele pode ser curado quando diagnosticado em sua fase inicial. A fisioterapia faz parte de uma equipe multidisciplinar de saúde e atua de forma bastante abrangente na sintomatologia dos pacientes oncológicos, tendo como principais objetivos sua reabilitação biopsicossocial e recuperação precoce da funcionalidade. Objetivos: Este estudo visou a descrever os métodos de avaliação e os recursos da fisioterapia oncológica e demonstrar seu reconhecimento por pacientes com câncer e por médicos nos hospitais públicos do Distrito Federal. Métodos: Foi feito um estudo transversal com aplicação de questionários a 30 fisioterapeutas, 44 pacientes e 45 médicos na rede hospitalar da Secretaria de Saúde. Resultados e Conclusão: Os resultados demonstraram que não há, na amostra pesquisada, a especialidade em fisioterapia oncológica, o que culmina com a falta de padronização da avaliação e do atendimento fisioterapêutico em pacientes com câncer. A maioria dos médicos desconhece os benefícios desse tratamento, não encaminhando seus pacientes aos fisioterapeutas, porém, mesmo não sendo encaminhados, os pacientes reconhecem a contribuição da fisioterapia para o seu tratamento.

Palavras-chave: Fisioterapia (Especialidade); Oncologia; Hospitais públicos; Avaliação de processos e resultados (Cuidados de Saúde)
\end{abstract}

Artigo científico apresentado como requisito parcial à conclusão do curso de Fisioterapia do Centro Universitário de Brasília (UniCEUB) em novembro de 2006.

${ }^{1}$ Fisioterapeutas graduados pelo UniCEUB, Brasília (DF), Brasil

${ }^{2}$ Fisioterapeuta graduada pelo UniCEUB, Brasília (DF), Brasil e Especialista em Fisioterapia Pneumofuncional pela Universidade de Brasília (UnB) ${ }^{3}$ Professora do UniCEUB, Brasília (DF), Brasil e Fisioterapeuta da Secretaria de Saúde do Distrito Federal (SES/DF). Orientadora da Pesquisa Endereço para correspondência: Caroline de Faria Silveira. Qd. Central - Conj. C - Bl. C - Apto 304 - Sobradinho - Brasília (DF), Brasil. CEP: 73010-703. E-mail: caroline_silveira@yahoo.com.br 


\section{INTRODUÇÃO}

Câncer é o nome dado a um grupo de doenças malignas caracterizadas pelo crescimento anormal e descontrolado de células que sofreram alteração em seu material genético, em algum momento de seu ciclo celular. Essas células geneticamente modificadas podem invadir os tecidos e órgãos, espalhando-se para outras regiôes do corpo ${ }^{1}$.

De acordo com os dados estatísticos da Organização Mundial da Saúde (OMS), são diagnosticados, por ano, 11 milhōes de casos de câncer no mundo. Os cânceres mais comuns entre homens e mulheres são os de pulmão, estômago e cólon retal. Nas mulheres, os tipos mais prevalentes são o câncer de mama e o do colo do útero. Já nos homens, o que mais prevalece é o câncer de pulmão e o câncer de estômago².

O Instituto Nacional de Câncer (INCA) fez uma estimativa para 2008 dos principais tipos de câncer que acometem a população brasileira. $\mathrm{O}$ número de casos de câncer de mama é de 51 casos novos para cada 100 mil mulheres. $\mathrm{O}$ número estimado de câncer de pulmão é de 19 casos para cada 100 mil homens e 10 casos para cada 100 mil mulheres. Para o câncer de estômago, o número é de 15 casos em 100 mil homens e 8 casos em 100 mil mulheres. A cada 100 mil mulheres, 19 desenvolvem câncer do colo do útero e, a cada 100 mil homens, 52 desenvolvem câncer de próstata ${ }^{3}$.

A estimativa no Distrito Federal, para o ano de 2008, também feita pelo INCA, demonstra que ocorrerão 5.620 novos casos de câncer, sendo 2.950 casos para o sexo feminino e 2.670 para o sexo masculino. O tipo de câncer que mais prevalece é o de mama, seguido pelos cânceres de próstata, cólon retal, pulmão e estômago ${ }^{4}$.

Segundo a OMS, o câncer é responsável por 12,5\% das mortes, por ano, em todo o mundo ${ }^{2}$. Estas estimativas parecem traduzir o estigma do sofrimento e morte que o câncer carrega consigo ${ }^{5}$, porém, quando diagnosticado em sua fase inicial, o câncer pode ser tratado por meio de cirurgia, radioterapia, hormonioterapia ou quimioterapia, aumentando muito a chance de cura do paciente ${ }^{6}$.

$\mathrm{O}$ diagnóstico do câncer implica não somente a descoberta das alteraçōes teciduais já instaladas, mas, muitas vezes, mudanças psíquicas e comportamentais naqueles que o carregam. Não raramente, o medo do tratamento, da quimioterapia e de todas as possíveis complicaçōes faz com que o paciente se afaste de seu meio e busque, inconscientemente, o isolamento ${ }^{5,7}$.

Somam-se às ocorrências descritas anteriormente as complicaçôes decorrentes da própria doença e de seu tratamento agressivo, tais como: fraqueza muscular, náuseas, vômitos, alteraçôes cardiovasculares e respiratórias, e as mais incapacitantes delas: a dor e a fadiga. Toda essa combinação pode levar aos sentimentos de depressão e angústia e causar piora do prognóstico do indivíduo ${ }^{5,8}$.

O tratamento oncológico deve ser instituído o mais precocemente possível e deve também ser definido de acordo com as situações clínicas apresentadas durante a evolução da doença, visando, assim, a uma melhor qualidade de vida do paciente. Diante disso, torna-se necessário promover um sistema de suporte ao paciente oncológico para que ele possa viver da forma mais ativa possível e sentir-se satisfeito em suas atividades?. Nesse contexto, a atuação multi e interdisciplinar contribui de maneira bastante efetiva para o sucesso do tratamento do câncer, já que consegue abordar as necessidades do indivíduo de forma específica e, ao mesmo tempo, global.

A fisioterapia é essencial nessa equipe por possuir um arsenal abrangente de técnicas que complementam os cuidados dos pacientes oncológicos, tanto na melhora da sintomatologia quanto da qualidade de vida, tendo como objetivos principais a reabilitação biopsicossocial e a recuperação precoce da funcionalidade do paciente?

A fisioterapia oncológica é uma especialidade que traz grandes benefícios para o tratamento de pacientes internados e especialmente para aqueles que fazem tratamento em domicílio. Seus recursos contribuem para complementar o alívio da dor, diminuir a tensão muscular, melhorar a circulação tecidual, prevenir ou reduzir linfedemas e minimizar a ansiedade do paciente, já que o estresse e a depressão podem ser agentes agravantes do câncer? ${ }^{9}$.

A escassez de dados na literatura e a falta de padronização de métodos de avaliação e de recursos a serem utilizados pela fisioterapia em pacientes oncológicos, bem como o desconhecimento dessa especialização entre os profissionais da saúde, tais como: médicos e fisioterapeutas, e também por parte da população, dificultam o acompanhamento fisioterapêutico, reduzindo, assim, a qualidade e a eficácia do tratamento contra o câncer.

Diante dessas dificuldades, o presente estudo visa a descrever os métodos de avaliação e os recursos da fisioterapia oncológica utilizados em hospitais públicos do Distrito Federal e demonstrar o seu reconhecimento por pacientes portadores de câncer e médicos no atendimento hospitalar.

\section{MATERIAIS E MÉTODOS}

Foi realizado um estudo transversal, utilizando uma amostra por conveniência de pacientes, fisioterapeutas e médicos da rede pública do Governo do Distrito 
Federal (GDF), utilizando questionários específicos (anexos I, II e III) para a coleta dos dados.

Os mesmos foram elaborados pelos autores, visto que na literatura não havia questionários pertinentes ao assunto. Para verificar a compreensão e a clareza das questôes, foi realizada previamente a aplicação de questionários pilotos a uma parte da amostra. Como não foram sugeridas alteraçôes, subentende-se que não houve dificuldade, por parte dos entrevistados, na resposta às perguntas, mantendo-se o questionário inicial.

Os questionários foram constituídos de perguntas abertas e fechadas. Nas questões abertas, objetivou-se permitir a exposição de opiniōes pessoais acerca do assunto questionado. Já as perguntas fechadas referiamse a questôes específicas, propiciando maior praticidade às respostas.

Os fisioterapeutas foram indagados sobre: sua especialização, se conhecem a especialidade de fisioterapia oncológica, se já atenderam pacientes com câncer e com qual freqüência; no caso de já terem atendido, foram questionados sobre os métodos fisioterapêuticos utilizados para avaliação e tratamento. Também foram questionados se o hospital oferece recursos suficientes para tratar tais pacientes e sobre qual a importância da fisioterapia nos pacientes portadores de neoplasias.

Os médicos foram questionados sobre a necessidade da atuação dos fisioterapeutas em pacientes oncológicos e se há contribuição para a melhora da qualidade de vida dos mesmos, bem como se encaminham rotineiramente esses pacientes ao serviço de fisioterapia.

Por fim, foi perguntado aos pacientes se eles receberam de seus médicos encaminhamento à fisioterapia, se eles estão realizando tratamento fisioterapêutico e, em caso positivo, o que estão achando. Além disso, os pacientes foram questionados sobre a contribuição da fisioterapia para seu tratamento.

Os 13 hospitais visitados foram: Hospital de Base do Distrito Federal (HBDF), Hospital de Apoio de Brasília (HAB), Hospital Regional da Asa Norte (HRAN), Hospital Regional da Asa Sul (HRAS), Hospital Regional de Taguatinga (HRT), Hospital São Vicente de Paulo (HSVP), Hospital Regional do Gama (HRG), Hospital Regional de Ceilândia (HRC), Hospital Regional de Samambaia (HRSam), Hospital Regional de Sobradinho (HRS), Hospital Regional do Paranoá (HRPa), Hospital Regional de Brazlândia (HRBz) e Hospital Dr. Hosannah Guimarães (antigo Hospital Regional de Planaltina - HRPl).

Os questionários foram aplicados, no período de 11 de setembro a 11 de outubro de 2006, pelos autores do artigo, sendo realizadas cerca de duas visitas a cada hospital em dias e horários convenientes aos pesquisadores. Esses questionários e os termos de consentimento foram entregues aos médicos e aos fisioterapeutas para serem respondidos e posteriormente recolhidos. Para os pacientes, o termo de consentimento e as perguntas do questionário foram lidos duas vezes, de modo imparcial, pelo pesquisador em forma de entrevista. Aduza-se que todos os entrevistados participaram voluntariamente desse projeto e assinaram um termo de consentimento livre e esclarecido, permitindo que os resultados obtidos fossem publicados.

Os critérios de inclusão para médicos e fisioterapeutas foram os seguintes: ser funcionário da rede hospitalar do GDF e estar presente no local nos dias das visitas pelos pesquisadores. Especificamente para os médicos, outro critério de inclusão é ter atendido pelo menos um paciente oncológico no hospital.

Para os pacientes, os critérios de inclusão foram ter diagnóstico clínico de câncer, estar consciente e orientado, e ter conhecimento de sua doença.

Foram usados como critérios de inclusão para os hospitais serem eles os maiores e mais conhecidos pela população do Distrito Federal. Já o critério de exclusão foi a não-concordância, por parte da direção, em realizar a pesquisa, ficando fora do projeto apenas um hospital dos previamente selecionados.

Os questionários foram analisados e interpretados separadamente, em três grupos específicos: o de fisioterapeutas, o de médicos e o de pacientes. Os resultados de cada grupo foram transformados em porcentagem de acordo com o número total de componentes para demonstrar as tendências dentro dos grupos pesquisados. Posteriormente, os gráficos dados foram gerados utilizando o programa SPSS - versão 12.0.

Por se tratar de pesquisas com seres humanos, o préprojeto foi submetido à avaliação e aprovação pelos Comitês de Ética em Pesquisa do Centro Universitário de Brasília (aprovação número 36/06) e da Secretaria de Saúde do Distrito Federal (aprovação número 740/2006).

\section{RESULTADOS}

A amostra total foi de 119 entrevistados, sendo 30 fisioterapeutas, 44 pacientes e 45 médicos.

Os resultados dos questionários aplicados aos fisioterapeutas demonstraram que $86,7 \%$ destes fizeram algum tipo de especialização, em áreas variadas, sendo elas: traumato-ortopedia $(30,2 \%)$, pneumofuncional $(20,9 \%)$, reeducação postural global $(11,6 \%)$, neurologia adulto, cardiologia e UTI adulto ( $7 \%$ cada), desportiva $(4,7 \%)$, neurologia infantil e adolescente, psicomotricidade, UTI neonatal e pediátrica, pilates e sistema locomotor $(2,3 \%$ cada). 
Em relação à especialidade de fisioterapia oncológica, $73,3 \%$ dos fisioterapeutas a conhecem. Dos 30 fisioterapeutas entrevistados, 56,7\% já atenderam pacientes com câncer na instituição. Entre estes, segundo as expectativas dos próprios fisioterapeutas, $64,7 \% \mathrm{o}$ fazem raramente, $23,5 \%$ sempre atendem e $11,8 \%$ quase sempre fazem atendimento a pacientes oncológicos.

De acordo com a figura 1, referente aos métodos de avaliação fisioterapêutica de pacientes oncológicos, a função pulmonar e a goniometria são utilizadas, cada uma, por $16,3 \%$ dos fisioterapeutas para avaliar os pacientes oncológicos. Já 18,4\% fazem avaliação da força muscular. Anamnese, escala da dor, análise da marcha e das atividades de vida diária (AVD) são utilizadas 6,1\%, cada. Questionários, inspeção, palpação, diagnóstico médico, prontuário, queixa principal, perda ponderal, avaliação cardíaca, avaliação ortopédica, tônus muscular, fadiga e edema são utilizados por $2 \%$ dos fisioterapeutas, cada.

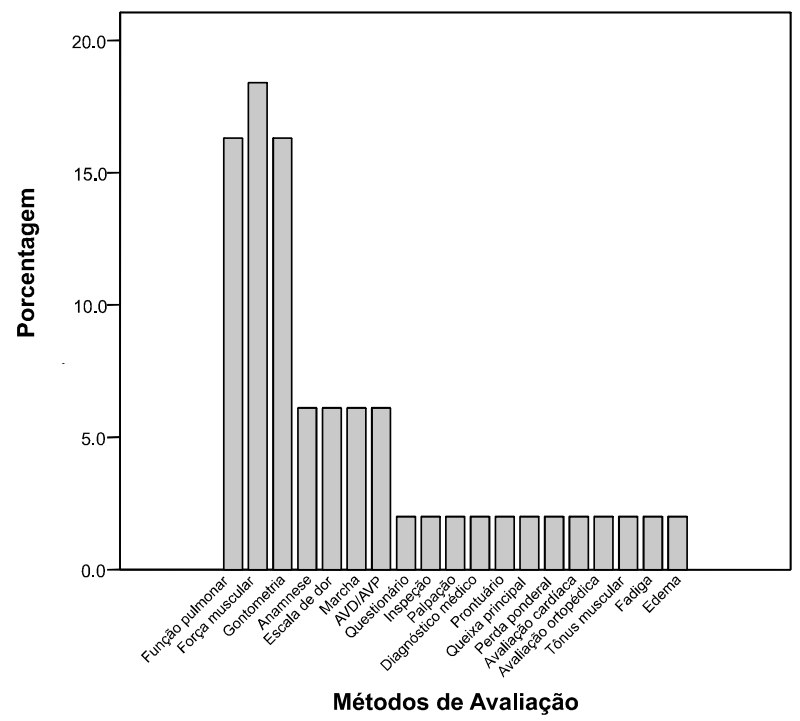

Figura 1. Métodos de avaliação fisioterapêutica de pacientes oncológicos

A figura 2 mostra que, no que diz respeito a recursos fisioterapêuticos utilizados em pacientes oncológicos, observou-se, a partir do questionário aplicado aos fisioterapeutas, que 3,7\% deles não utilizam qualquer recurso eletrofototermoterapêutico (EFT) para tratar seus pacientes oncológicos; $2,8 \%$ utilizam crioterapia e estimulação elétrica nervosa transcutânea, cada; 4,6\% utilizam outros recursos elétricos, sendo eles: calor superficial, ultra-som, compressão pneumática, estimulação elétrica funcional e ventilação mecânica.

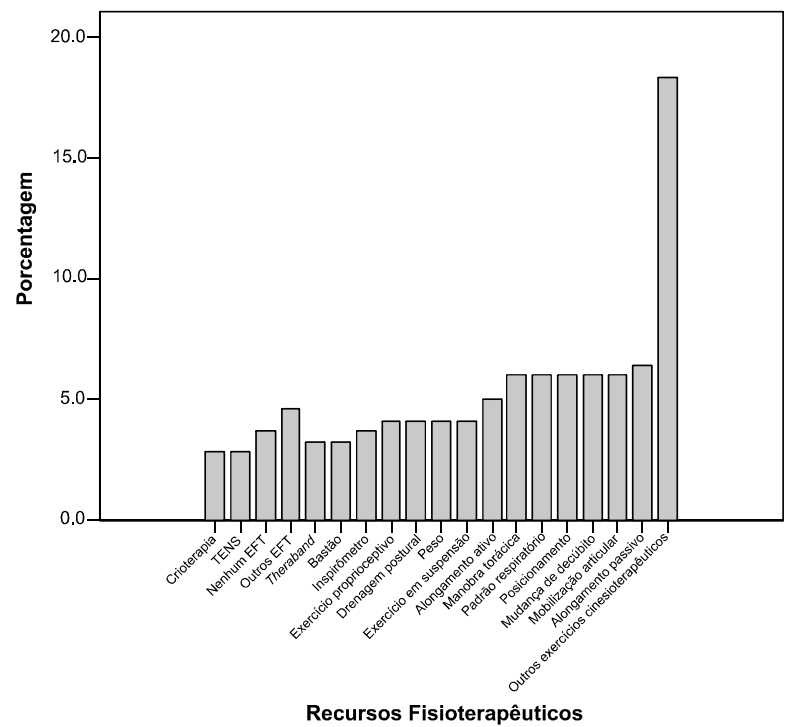

Figura 2. Recursos fisioterapêuticos utilizados em pacientes oncológicos

A mesma figura demonstra que os alongamentos passivo e ativo são usados, respectivamente, por $6,4 \%$ e 5,0\% dos fisioterapeutas. Mudança de decúbito, mobilização articular, posicionamento no leito, padrões respiratórios e manobras torácicas são utilizados 6,0\%, cada. Exercícios em suspensão, pesos, drenagem postural e exercícios proprioceptivos são usados $4,1 \%$, cada; incentivador de fluxo $3,7 \%$; bastão e theraband $3,2 \%$, cada. Além disso, 18,3\% dos fisioterapeutas utilizam outros recursos fisioterapêuticos, tais como: massagens relaxantes, estimulantes e demais tipos, exercícios de Codmam, bandagem compressiva, Kabat, Bobath, Mackenzie, pilates, flutter, acapella, reeducação postural global, bola suíça, iso-stretching, e exercícios de Nicolas.

De acordo com a pesquisa, $73,3 \%$ dos fisioterapeutas alegaram que os hospitais não oferecem recursos fisioterapêuticos suficientes para tratar os pacientes oncológicos e 6,7\% não souberam relatar tal situação.

Dos fisioterapeutas entrevistados, $23 \%$ acreditam que o tratamento melhora a qualidade de vida dos pacientes; $13,5 \%$ afirmam que melhora a funcionalidade e independência nas AVD; $12,2 \%$ alegam que há melhora da função cardiopulmonar, $12,2 \%$ disseram melhorar a função musculoesquelética; 10,8\% referem o alívio do quadro álgico; $9,5 \%$ disseram que a fisioterapia atua na prevenção e no tratamento de complicaçōes; 9,5\% disseram que preparam e orientam os pacientes para todo o tratamento farmacológico; $5,4 \%$ disseram que há diminuição do tempo de internação e $4,1 \%$ mencionam que o tratamento fisioterapêutico contribui para aumentar a sobrevida dos pacientes, como mostra a figura 3 . 


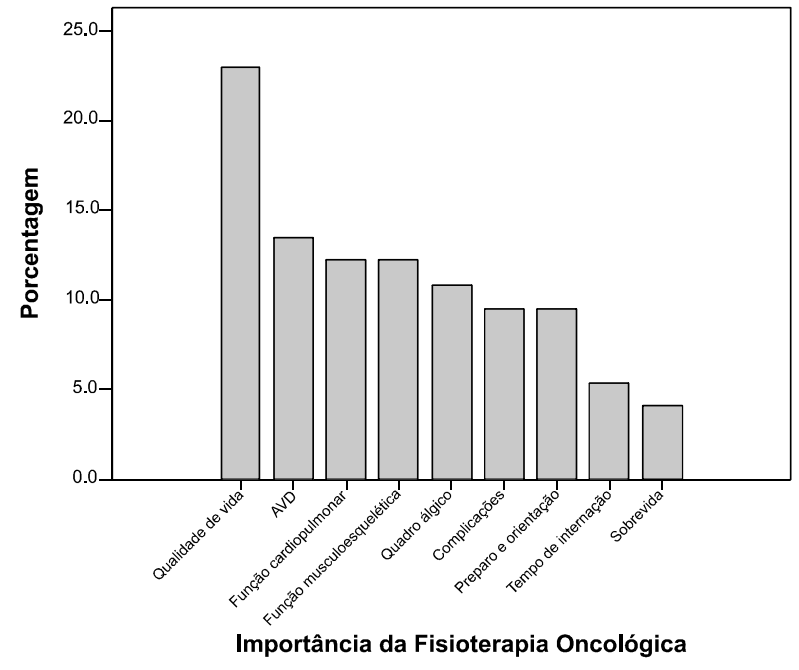

Figura 3. Importância da fisioterapia em pacientes oncológicos, de acordo com fisioterapeutas

Ainda na mesma questão, dezesseis fisioterapeutas tiveram uma visão geral acerca da importância da fisioterapia em pacientes oncológicos: 18,8\% deles ressaltaram a necessidade de tratamento paliativo, 18,8\% acreditam que a fisioterapia depende do quadro geral do paciente e $18,8 \%$ referem que o tratamento pode ser feito em qualquer quadro clínico. Além disso, 43,8\% deles a consideram essencial nos cuidados desses pacientes.

Com relação aos médicos, 55,6\% deles conhecem a atuação da fisioterapia no tratamento de pacientes oncológicos. Dos 45 entrevistados, 97,8\% afirmaram que o tratamento fisioterapêutico contribui para a melhora na qualidade de vida desses pacientes, enquanto $2,2 \%$ relataram não saber a respeito de tal contribuição. A fisioterapia é considerada necessária ao tratamento dos pacientes oncológicos por $51,1 \%$ dos médicos, enquanto $46,7 \%$ deles a consideram muito necessária e 2,2\% pouco necessária.

Entre os resultados esperados do tratamento fisioterapêutico nos pacientes com câncer, na visão dos médicos, as respostas citadas foram: melhora da qualidade de vida $(30,4 \%)$, melhora da função musculoesquelética $(28,3 \%)$, melhora da função respiratória $(18,5 \%)$, prevenção e tratamento de complicaçōes $(9,8 \%)$, analgesia $(7,6 \%)$, diminuição do tempo de internação, aumento da tolerância ao tratamento, reabilitação precoce do paciente, melhora da capacidade laborativa e complemento ao tratamento clínico $(1,1 \%$ cada), como descreve a figura 4 .

$\mathrm{O}$ encaminhamento de rotina de pacientes para a fisioterapia é feito por $46,7 \%$ dos médicos. Os demais justificam o não-encaminhamento por desconhecimento (36,0\%), ausência do serviço no hospital $(24,0 \%)$,

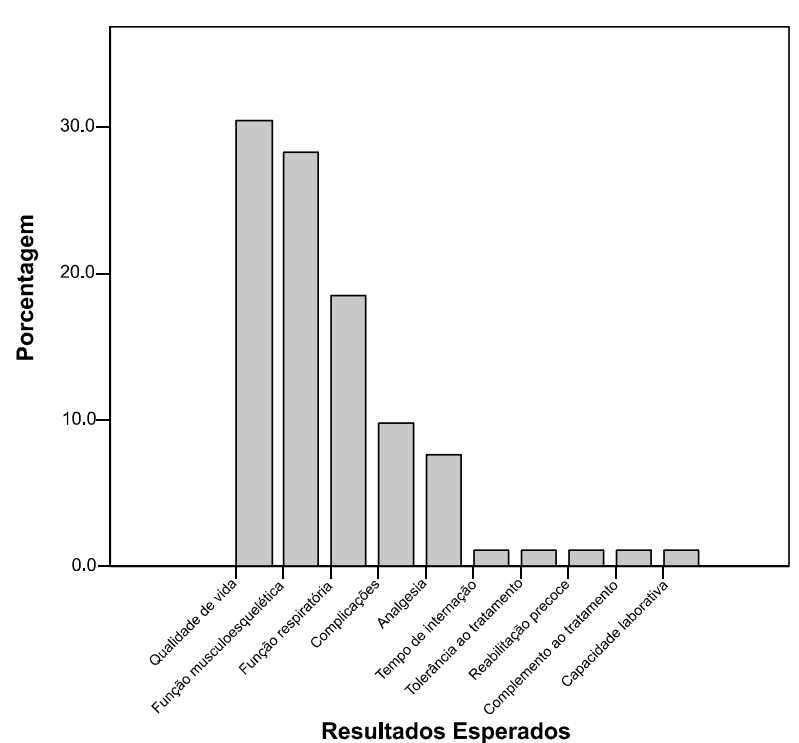

Figura 4. Resultados esperados do tratamento fisioterapêutico em pacientes oncológicos por médicos

encaminhamento do paciente para outra regional mais especializada e falta de fisioterapeuta na instituição (12,0\% cada), falta de condição física e dependência da patologia do paciente ( $4,0 \%$ cada).

Entre os 44 pacientes entrevistados, 90,9\% estavam internados nos hospitais em que as entrevistas foram feitas, $54,5 \%$ sabem o que é fisioterapia e $72,7 \%$ não foram encaminhados para o tratamento fisioterapêutico por seus médicos.

De acordo com os resultados obtidos, entre os pacientes que recebem acompanhamento fisioterapêutico, $58,3 \%$ classificaram o tratamento como bom, $25 \%$, como ótimo e $16,7 \%$, como excelente.

Constatou-se, ainda, que $63,6 \%$ dos pacientes afirmam que a fisioterapia pode contribuir para seu tratamento, enquanto $20,5 \%$ mencionam que o tratamento fisioterapêutico não contribui e $15,9 \%$ não sabem informar.

\section{DISCUSSÃO}

Com base nos resultados dos questionários aplicados aos fisioterapeutas, observou-se que a maioria deles apresenta especialização em pelo menos uma área, sendo as mais citadas traumato-ortopédica e pneumofuncional. Essa constatação corrobora a imagem da fisioterapia há alguns anos, a qual estava vinculada apenas à ortopedia e à pneumologia. Entretanto, hoje, o fisioterapeuta atua em todas as áreas da saúde.

Apesar de a maioria dos fisioterapeutas dos hospitais públicos do GDF conhecer a especialidade de fisioterapia 
oncológica, verificou-se que $26,7 \%$ dos entrevistados ainda a desconhecem. Essa informação pode estar relacionada tanto ao fato de essa área não ser bem divulgada entre os profissionais da saúde como, provavelmente, por não existirem cursos de especialização em fisioterapia oncológica no Distrito Federal ou em áreas do entorno. Somam-se a isso os fatos de ser uma especialidade nova e ainda não reconhecida pelo Conselho Federal de Fisioterapia e Terapia Ocupacional (COFFITO).

Grande parte dos fisioterapeutas entrevistados raramente atende a pacientes oncológicos nos hospitais públicos, fato que pode ser atribuído à pouca divulgação da especialidade em oncologia e até mesmo ao seu desconhecimento entre os profissionais da saúde.

$\mathrm{O}$ atendimento aos pacientes com câncer pelos fisioterapeutas é fundamental durante todas as fases de tratamento, porque esse profissional oferece acompanhamento às diversas alterações e complicaçōes que podem surgir durante esse período, tais como: edema de membros, alterações respiratórias, alterações circulatórias e vasculares, dentre outras ${ }^{7}$.

Acrescenta-se que a fisioterapia tem uma atuação essencial dentro da oncologia. Ela não se preocupa apenas com o local afetado pelo câncer, mas com todo o indivíduo e principalmente com sua qualidade de vida ${ }^{10}$.

Em relação aos métodos de avaliação usados pelos fisioterapeutas em pacientes oncológicos, parece haver uma grande variedade de opções. Os mais citados foram força muscular, função pulmonar e goniometria, possivelmente por constituírem os dados básicos de qualquer avaliação fisioterapêutica. Outros métodos menos citados, mas que apresentam grande importância em uma avaliação específica de pacientes com câncer, são dor e fadiga. Segundo Pimenta e Mota, apesar de esses sintomas estarem presentes em $2 / 3$ dos pacientes oncológicos, os profissionais de saúde não têm conhecimento sobre como avaliá-los, tendo em vista serem eles subjetivos e multifatoriais ${ }^{9,11}$.

Os recursos utilizados pelos fisioterapeutas entrevistados também somam uma grande variedade de opções para o tratamento de pacientes oncológicos. A opção por esses recursos depende muito do tipo de câncer, das complicações e dos sintomas que o paciente apresenta ${ }^{12}$. Além disso, a maioria dos fisioterapeutas alega que os hospitais não oferecem recursos suficientes para tratar esses pacientes. Uma hipótese para justificar esses achados seria o próprio desconhecimento da atuação em oncologia por parte dos fisioterapeutas, a qual deve ser testada em outros estudos.

Os fisioterapeutas entrevistados apresentaram diferentes opiniōes em relação à importância da fisioterapia em pacientes oncológicos, mas todas elas culminam para um objetivo primário que é melhorar a qualidade de vida desses pacientes, por meio, principalmente, do resgate de sua funcionalidade e de sua independência. $\mathrm{O}$ alcance desse objetivo independe do quadro clínico geral do paciente ${ }^{13}$, diferentemente do que foi citado por alguns desses profissionais. Os pacientes com câncer, principalmente aqueles fora da possibilidade de cura, necessitam ainda mais da abordagem humanista dos profissionais que atuam em seu tratamento. Essa ideologia de cuidar do paciente em todos os aspectos - físico, espiritual, psicológico e social - e em todas as fases de sua doença constitui a base dos cuidados paliativos ${ }^{12,14}$ também citados por diversos entrevistados.

Entre os médicos entrevistados, a maioria afirmou conhecer a atuação da fisioterapia oncológica, bem como sua contribuição na melhora da qualidade de vida dos pacientes com câncer. No entanto, pouco mais da metade acha apenas necessário o tratamento fisioterapêutico nesses pacientes.

Com relação aos resultados esperados, a melhora da qualidade de vida foi um dos itens mais citados tanto por médicos quanto por fisioterapeutas, porém esse é um conceito muito amplo no que se refere aos objetivos do tratamento fisioterapêutico, visto que a qualidade de vida é almejada por todos os profissionais da saúde que compóem uma equipe multidisciplinar. Diante disso, sua simples referência como meta esperada do tratamento parece não implicar o conhecimento da atuação fisioterapêutica em pacientes oncológicos.

O desconhecimento por parte da maioria dos médicos dos benefícios proporcionados pela fisioterapia oncológica e a falta desse serviço nos hospitais públicos são os principais fatores que contribuem para o nãoencaminhamento dos pacientes com câncer para o tratamento fisioterapêutico.

Aventa-se a hipótese de que, por ser uma especialidade relativamente nova dentro da fisioterapia e por haver escassez de pesquisas científicas nessa área, os próprios fisioterapeutas desconhecem sua possível atuação em pacientes oncológicos. Conseqüentemente, sua divulgação entre os profissionais da saúde torna-se deficiente, culminando na falta desse serviço nos hospitais.

Todos os pacientes oncológicos que têm acesso ao tratamento fisioterapêutico reconhecem os seus benefícios. Mesmo os pacientes que não têm esse acompanhamento mencionam que a fisioterapia pode contribuir para o seu tratamento, o que demonstra o reconhecimento desse serviço por essa população. 


\section{CONCLUSÃO}

O presente estudo constatou a realidade do serviço de fisioterapia oncológica nos hospitais públicos do Distrito Federal. Os métodos de avaliação e os recursos utilizados pelos fisioterapeutas nesses hospitais são divergentes, tendo em vista não existir ainda um padrão para tratamento de pacientes oncológicos.

Constatou-se, por fim, que os médicos não encaminham pacientes com câncer para tratamento com os fisioterapeutas pela falta desse serviço nos hospitais e pelo próprio desconhecimento dos benefícios proporcionados por essa terapia. Ademais, os pacientes oncológicos reconhecem que o tratamento fisioterapêutico pode contribuir para a melhora de seu quadro clínico geral.

\section{REFERÊNCIAS}

1. Robbins SL, Cotran RS, Kumar V. Neoplasias. In: Robbins SL, Cotran RS, Kumar V. Patologia estrutural e funcional. 5a ed. Rio de Janeiro: Guanabara Koogan; 1996. p. 213-68.

2. World Health Organization [homepage on the Internet]. Geneva: World Health Organization; c2008 [cited 2006 Out 23]. Cancer; [about 2 screeens]. Available from: http:/ /www.who.int/cancer/en/.

3. Instituto Nacional de Câncer. Estimativa 2008: incidência de câncer no Brasil [monografia na Internet]. Rio de Janeiro (Brasil): Instituto Nacional de Câncer; 2008 [citado em 2008 Set 24]. Disponível em: http://www.inca.gov.br/ estimativa $/ 2008 /$ index.asp? link =conteudo_ view.asp\&ID=5.

4. Instituto Nacional de Câncer. Estimativa 2008: incidência de câncer no Brasil [monografia na Internet]. Rio de Janeiro
(Brasil): Instituto Nacional de Câncer; 2008 [citado em 2008 Set 29]. Disponível em: http://www.inca.gov.br/ estimativa/2008/index.asp?link=tabelaestados . asp\&UF=DF.

5. Bloom JR, Kessler L. Emotional support following cancer: a test of the stigma and social activity hypotheses. J Health Soc Behav. 1994;35(2):118-33.

6. Instituto do Câncer Dr. Arnaldo [homepage na Internet]. São Paulo: Instituto do Câncer Dr. Arnaldo; c2008 [citado em 2006 Out 24]. Disponível em: http:// www.icavc.com.br/materia.php?ref=oqueeca.

7. Friedrich CF, Souza RV, Ruiz SAL, Denari SC. O papel do fisioterapeuta no tratamento oncológico. In: Baracat FF, Fernandes HJJ, Silva MJ. Cancerologia atual: um enfoque multidisciplinar. São Paulo: Roca; 2000. p. 198-204.

8. Mota DDCF, Pimenta CAM. Fadiga em pacientes com câncer avançado: conceito, avaliação e intervenção. Rev Bras Cancerol. 2002;48(4):577-83.

9. Pimenta CAM. Dor oncológica: bases para avaliação e tratamento. Mundo Saúde. 2003;27(1):98-110.

10. Kelly S. Tratamento paliativo no câncer. In: Spence RAJ, Johnston PG, editores. Oncologia. Rio de Janeiro: Guanabara Koogan; 2003. p. 107-22.

11. Mota DDCF, Pimenta CAM. Fadiga em pacientes recebendo cuidados paliativos. Mundo Saúde. 2003;27(1):111-7.

12. Pessini L, Caponero R, Melo AGC. Cuidados paliativos: uma necessidade urgente na área da saúde. Mundo Saúde. 2003;27(1):3-5.

13. Marcucci FCI. O papel da fisioterapia nos cuidados paliativos a pacientes com câncer. Rev Bras Cancerol. 2005;51(1):67-77.

14. Santiago-Palma J, Payne R. Palliative care and rehabilitation. Cancer. 2001:92 Suppl 4:S1049-52. 


\section{QUESTIONÁRIO PARA O FISIOTERAPEUTA}

\section{INSTRUÇÕES}

Esta pesquisa visa a verificar os métodos de avaliação e os recursos fisioterapêuticos na área de oncologia utilizados nos hospitais públicos do Distrito Federal. Por favor, responda aos itens abaixo evitando deixar Respostas em branco.

Este questionário não permite a identificação do indivíduo que o respondeu. Obrigado.

1- Você possui alguma especialização?
( ) Sim
( ) Não

2- Se sim, qual?
( ) Traumato-ortopedia
( ) Neurologia adulto
( ) Hidroterapia
( ) Neurologia infantil / adolescente
( ) Cardiologia
( ) Pneumofuncional
( ) Dermatofuncional
( ) Uro - ginecologia e Obstetrícia
( ) Desportiva
( ) Fisioterapia do Trabalho
( ) Geriatria
( ) UTI - adulto
( ) Psicomotricidade
( ) UTI - neonatal e pediátrica
( ) Oncologia
( ) Outros:

3- Você conhece a especialidade fisioterapia oncológica?
( ) Sim
( ) Não

4- Você já atendeu a pacientes oncológicos nesta instituição?
( ) Sim
( ) Não

5- Se sim, com que freqüência você atende a pacientes oncológicos?
( ) Sempre
( ) Quase sempre
( ) Raramente

6- Se já tiver atendido, quais os métodos que você utilizou para avaliar esse(s) paciente(s)? 
7- Neste caso, quais recursos eletrofototermoterapêuticos você utilizou?
( ) TENS
( ) Ultra-som
( ) FES
( ) Crioterapia
( ) Calor superficial
( ) Ondas curtas
( ) Correntes elétricas
( ) Radiação ultravioleta
( ) Turbilhão
( ) Compressão pneumática
( ) Laser
( ) Microondas
( ) Outros:

8- Nesses pacientes, quais outros recursos você utilizou?
( ) Massagem relaxante
( ) Quiropraxia
( ) Massagem estimulante
( ) Pilates
( ) Outras massagens
( ) RPG
( ) Osteopatia
( ) Iso-stretching
( ) Acupuntura
( ) Kabat (PNF)
( ) Bobath
( ) Mackenzie
( ) Bola suíça
( ) Exercícios de Codmam
( ) Alongamento passivo
( ) Exercícios de Nicolas
( ) Alongamento ativo
( ) Mudança de decúbito
( ) Mobilização articular
( ) Posicionamento no leito
( ) Pesos
( ) Padrōes respiratórios
( ) Bastão
( ) Exercícios em suspensão
( ) Theraband
( ) Bandagem compressiva
( ) Manobras torácicas
( ) Drenagem linfática
( ) CPAP
( ) Flutter
( ) Acapella
( ) Incentivador de fluxo
( ) Threshold
( ) Exercícios proprioceptivos
( ) Outros:

9- A instituição oferece recursos fisioterapêuticos suficientes para um tratamento adequado dos pacientes oncológicos?
( ) $\operatorname{Sim}$
( ) Não

10- Na sua opinião, qual a importância da fisioterapia em pacientes oncológicos? 
ANEXO II

\section{QUESTIONÁRIO PARA O MÉDICO}

\section{INSTRUÇÕES}

Essa pesquisa visa a avaliar o reconhecimento da fisioterapia no tratamento de pacientes oncológicos. Por favor, responda aos itens abaixo evitando deixar respostas em branco.

Este questionário não permite a identificação do indivíduo que o respondeu. Obrigado.

1- Você conhece a atuação da fisioterapia no tratamento de pacientes oncológicos?
( ) $\operatorname{Sim}$
( ) Não

2- Você acha que o tratamento fisioterapêutico contribui para a melhora da qualidade de vida do paciente oncológico?
( ) $\operatorname{Sim}$
( ) Não

3- Como você vê o tratamento fisioterapêutico em pacientes oncológicos?
( ) Desnecessário
( ) Pouco necessário
( ) Necessário
( ) Muito necessário

4- Quais resultados você espera do tratamento fisioterapêutico em pacientes oncológicos?

5- Você encaminha, de rotina, seus pacientes para tratamento fisioterapêutico?
( ) $\operatorname{Sim}$
( ) Não

6- Se não, por quê? 
ANEXO III

\section{QUESTIONÁRIO PARA O PACIENTE}

\section{INSTRUÇÕES}

Esta pesquisa visa a avaliar o reconhecimento da fisioterapia no tratamento de pacientes com câncer. Por favor, responda aos itens abaixo evitando deixar respostas em branco.

Este questionário não permite a identificação do indivíduo que o respondeu. Obrigado.

1- Você está internado neste hospital?

$$
\text { ( ) Sim ( ) Não }
$$

2- Você sabe o que é o tratamento fisioterapêutico?
( ) Sim
( ) Não

3- O seu médico indicou acompanhamento fisioterapêutico durante seu tratamento?
( ) Sim
( ) Não

4- Você está fazendo fisioterapia?
( ) Sim
( ) Não

5- Se sim, o que você está achando do tratamento?
( ) Excelente
( ) Ótimo
( ) Bom
( ) Ruim
( ) Péssimo

6- Você acha que a fisioterapia pode contribuir para o seu tratamento?
( ) Sim
( ) Não 


\section{Abstract}

Introduction: Cancer is the name given to a group of malignant diseases characterized by unusual cell growth and which can spread to various regions of the body. Although it is annually responsible for $12.5 \%$ of deaths worldwide, it can be cured when diagnosed in its initial stages. Physiotherapy is part of a multidisciplinary health team that can respond to many of the oncologic patients' symptoms, having as its main purpose the patients' bio-psycho-social regeneration and the early recovery of functionality. Objective: This study aimed at describing the evaluation methods and the oncologic physiotherapy resources and at showing their recognition by patients with cancer and by doctors in public hospitals in the Federal District of Brazil. Methods: Questionnaires were filled out by 30 physiotherapists, 44 patients and 45 doctors in the public hospital network from the Secretary of Health in a transversal study. Results and Conclusion: The results showed that, in the sample studied, a specialization in oncologic physiotherapy does not exist, which results in a lack of standardization of physiotherapeutic evaluation and service for cancer patients. The majority of the doctors are not aware of the benefits associated with this treatment and do not indicate their patients to physiotherapists. However, even though the patients are not sent to physiotherapy, they do recognize that physiotherapy could contribute to their treatment.

Key words: Physical therapy (Specialty); Medical oncology; Hospitals, public; Outcome and process assessment (Health Care) 